\title{
Variability in species abundance can drive flower diversification and specialization
}

\author{
Sébastien Rivest ${ }^{1}$ \\ ${ }^{1}$ University of Ottawa
}

September 21, 2020

\begin{abstract}
Angiosperms show remarkable floral diversity. However, the ecological processes involved in flower diversification remain poorly understood. In this article I propose that different plant species abundance drives adaptation to different pollinators and promotes different degrees of specialization. In this view, interspecific variation in species abundance can foster floral diversification. I develop a mathematical model of pollen transfer considering the interaction of several pollination processespollen removal and carryover, intra- and interspecific competition for pollinator visitation, and interspecific pollen transfer- that are linked to floral abundance. To assess if and how floral abundance can generate floral diversity, I use the model to assemble plant-pollinator networks from simulated plant and pollinator communities. The model shows that evolution of flowers towards highly specialized pollinators and pollinators with high pollen carryover capacity is favoured at low floral abundance, while evolution on more abundant pollinators is favoured at higher abundance. Furthermore, floral specialization is favoured at low floral abundance, while generalization is favoured at high abundance. In simulated plant communities of variable floral abundance, different pollinator systems evolve among the different plant species. The model demonstrates a new mechanism by which floral diversity can be generated, contributing to our understanding of floral evolution and diversification.
\end{abstract}

\section{Introduction}

A defining feature of the angiosperms is their remarkable floral diversity. (Armbruster 2014; HernándezHernández \& Wiens 2020). Darwin first recognized that the diversity of floral shape, size, colour and scent could be attributable to selection by pollinators (Darwin 1877). This realization has resulted in a large modern research program, spawning ecological, evolutionary and genetic studies, investigating how interactions between plants and pollinators drive floral evolution (Harder \& Johnson 2009; Van der Niet \& Johnson 2012). While we are now heading toward a strong mechanistic understanding of how flowers evolve (Moyroud \& Glover 2017; Shan et al. 2019; Fattorini \& Glover 2020), the ecological processes involved in flower diversification remain poorly understood (Kay \& Sargent 2009; Johnson 2010; Van der Niet \& Johnson 2012; Armbruster 2017).

Most of our understanding of how flowers diversify derives from two principles, which were combined into the Grant-Stebbins model (Johnson 2006, 2010). First, flowering plants should adapt to the most effective pollinator in a given environment (Stebbins 1970); that is, the pollinator that visits most frequently (number of visits) and efficiently (per-visit pollen transport efficiency) (Armbruster 2014). Second, since pollinator assemblages are geographically variable, plants should be under divergent selection in different environments, resulting in adaptation to different pollinators (Grant \& Grant 1965).

Shifts in floral characteristics and pollination systems, however, frequently occur without associated geographical shifts in pollinator assemblages, suggesting that other factors drive flower diversification (Herrera et al. 2006; Van Der Niet et al. 2014; Ferreiroet al. 2017). In the last few decades, several studies have emphasized the importance of community context in understanding the ecology and evolution of plant pol- 
lination (Caruso 2000; Sargent \& Ackerly 2008; Mitchell et al. 2009; Muchhala et al. 2010). Competition and facilitation among plant species for access to pollinator visitation, and interspecific pollen transfer play important roles in determining the outcome of pollination (Geber \& Moeller 2006; Morales \& Traveset 2008; Sargent \& Ackerly 2008; Mitchell et al. 2009; Pauw 2013). Furthermore, competition via interspecific pollen transfer offers a potential mechanism promoting divergence in pollinator use by favoring reduced pollinator sharing (Muchhala et al. 2010; Moreira-Hernández \& Muchhala 2019). However, despite now having a more comprehensive understanding of pollination ecology and several hypotheses having been proposed to explain either variability in pollinator use or degree of specialization (Waser et al. 1996; Johnson \& Steiner 2000; Aigner 2001; Gómez \& Regino 2006; Sargent \& Otto 2006; Muchhala et al. 2010; Moreira-Hernández \& Muchhala 2019), we still lack a theory explaining the broad patterns of floral diversity within and among communities.

A general mechanism promoting floral diversity in both pollinator use and degree of specialization might derive from the consideration that several processes governing pollination success (e.g. intraspecific competition for access to pollinator visitation, interspecific pollen transfer and pollen carryover) are modulated by floral abundance, which intrinsically varies among species within communities. It is therefore possible that different plant species of different floral abundances face divergent selective pressures from a same pollinator assemblage. For example, interspecific pollen transfer is expected to have a stronger impact on populations of low abundance, as the proportion of interspecific pollinator visits increases with the proportion of heterospecific individuals in plant communities (Rathcke 1983; Feinsinger et al. 1991; Caruso 2002; Palmer et al. 2003; Sargent \& Otto 2006; Mitchell et al. 2009; Runquist \& Stanton 2013). Likewise, opportunities for pollen loss, whether passively or due to pollinator grooming, should be greater for rare plant species because pollinators visiting rare plants spend more time between conspecific visits (Minnaar et al. 2019). Therefore, pollen carryover - the proportion of the removed pollen carried to the next conspecific flower - is expected to increase with floral abundance. Conversely, intraspecific competition for pollinator visitation should be stronger at high floral abundance, as more flowers compete for visitation by the same pollinator community (Rathcke 1983; Geber \& Moeller 2006; Duffy \& Stout 2008; Pauw 2013; Benadi \& Pauw 2018). While studies carried out at limited spatial scales often find increased per flower pollinator visitation with increasing floral density (due to greater attractiveness of larger flowering patches) (see Ghazoul 2005), at the landscape scale - the scale that ultimately matters for floral evolution - the opposite trend is observed: the number of visits received per flower decreases with a species abundance (see Pauw 2013 and references therein; Hegland 2014; Benadi \& Pauw 2018; Bergamo et al. 2020). The reason for such scale dependency is simple: large floral patches are more attractive to pollinators, but a population composed of multiple large floral patches will saturate the pollinators available, leading to stronger intraspecific competition.

In this article, I propose that the pollination system offering the optimal evolutionary solution for a plant species is a function of the plant's relative abundance in a community. In this view, different pollinators and degrees of specialization are favoured at different floral abundances. Floral diversification can result from shifts in relative species abundance associated with the colonization of new habitats or geographical ranges, creating new conditions under which floral diversification can occur. Abundance has been previously identified as a potential driver of floral specialization (Feinsinger 1983; Sargent \& Otto 2006). However, the potential for variability in species abundance to drive adaptation to different types of pollinators has never been considered before. To demonstrate the potential of variability in species abundance to foster floral diversity, I develop a simple mathematical model of pollen transfer considering the interaction of several pollination processespollen carryover, pollen removal, intra- and interspecific competition for pollinator visits, and interspecific pollen transfer. By tracking pollen fate, the model explicitly measures male reproductive success. Female reproductive success should be affected similarly to male reproductive success as long as increased conspecific pollen deposition to stigmas increases seed production. I use the model to assemble plant-pollinator networks from simulated plant and pollinator communities. In a community context, the interactions between plants and their pollinators are generally investigated in terms of networks of interactions. Using this approach, I assess if and how interspecific variation in floral abundance generates diversity in pollinator use and degree of specialization. In addition to supporting the conceptual model, the mathematical model is consistent 
with, and suggests explanations for, several patterns governing the evolution, diversification and community assembly of flowers.

\section{How floral abundance drives flower diversification: a conceptual model}

Since plants produce a finite number of gametes, optimizing reproductive success requires maximizing the number of ovules fertilized by a finite amount of pollen. When limited by pollinator quantity, plants should benefit from being less restrictive in their flower accessibility. Any visitor, regardless of its quality (pollen carryover efficiency and specialization), is likely to increase the number of pollen grains deposited on conspecific stigmas (Thomson et al. 2000; Thomson 2003; Muchhala et al. 2010). However, pollen grains have a higher probability of reaching conspecific stigmas when carried by more efficient pollinators. When enough pollinators are available to remove most pollen grains, pollinator quality becomes more limiting to plant reproductive success than pollinator quantity, and plants should specialize on the most efficient pollinator (Thomson et al. 2000; Thomson 2003).

Here I propose that the selective importance of pollinator quantity and quality is modulated by floral abundance. At high floral abundance, as more pollinators are required for sufficient pollination, plant reproduction is more strongly limited by pollinator quantity. Conversely, pollen loss to inefficient carryover and interspecific visits is reduced as floral density increases. Therefore, increased floral abundance should increase the selective importance of pollinator quantity while reducing the selective importance of pollinator quality. Under these conditions, plants should benefit from generalized pollination where more pollinators, but perhaps more wasteful ones, have access to flowers. At low abundance, the dynamic is reversed and plants should benefit from specializing on efficient carriers of their pollen.

While I have so far considered a dichotomy between low and high abundance, plant populations can exist in any state from extremely rare to abundant. Likewise, pollinators vary in their abundance (quantity component) and efficiency (quality component). As each pollinator offers a unique combination of quantity and quality components of pollination, changes in a plant species' relative abundance in a community will shift the identity of the pollinator representing the most effective pollinator, and therefore, the pollination system resulting in a fitness optimum. In this view, floral abundance shapes the plant selective landscape. Variations in plant abundance move the fitness peak of the selective landscape toward different pollination systems, fostering floral diversification.

\section{Model of pollen transfer}

Here I develop a mathematical model which determines how the optimal pollinator or set of pollinators for a plant population changes as a function of floral abundance. The model measures the proportion of pollen grains produced by a single flower of the focal species (hereafter 'focal flower') that reaches conspecific stigmas. This value is influenced by the interaction of several pollination processes that are linked to floral abundance (described above): pollen removal and carryover, intra- and interspecific competition for pollinator visitation, and interspecific pollen transfer. By tracking pollen fate, the mathematical model explicitly determines the pollination system maximizing male reproductive success. However, selection through either male and female function is expected to reach the same solution in terms of optimal pollination system as long as pollen receipt and export are limiting and are governed by the same variables for each sex (i.e. pollinator identity and abundance).

In the model, I treat pollinators as functional groups of pollinator species with similar attributes (morphology, behaviour) that produce similar selection on flowers (e.g. different species of hummingbirds, large-bodied bees) (Fenster et al. 2004). The model assumes that flowers are distributed homogeneously in space (i.e., flowers of the same species are not more likely to be near one another). Variation in floral abundance in the model is therefore the result of variation in the abundance of the focal plant species rather than variation in the number of flowers produced per individual of the focal species. By assuming homogeneous spatial distribution, the model does not consider the potential for facilitation among species sharing the 
same pollinators, although facilitation is expected to benefit rarer species (Rathcke 1983; Steven et al. 2003; Essenberg 2012), contributing to the predicted increase in quantity limitation with abundance -i.e. facilitation should reinforce the predictions from the conceptual model.

For a given plant species $a$, the total number of pollen grains produced per flower is represented by $P_{t}$ . The proportion of grains removed with each pollinator visit to a focal flower is represented by $R_{i}$, and depends on both the attributes of the pollinator $i$ and of the focal plant species. While adaptation toward a pollination system could, theoretically, increase $R$, I am more interested in the causes of shifts between pollination systems rather the mechanisms leading to a subsequent better fit to the system, so the model does not consider evolution of $R_{i}$. With each new visit to a focal flower, the amount of pollen remaining on the anthers diminishes, and the amount of pollen picked up with each new pollinator visit diminishes proportionally to the number of visits already received, resulting in an exponential decline of pollen removed with each new visit (Young \& Stanton 1990; Robertson \& Lloyd 1993). Considering that pollinator $i$ is the only visitor (floral generalization is considered in equation 5), the total amount of pollen removed, $P_{r}$, from the focal flower by a given pollinator $i$ is therefore

$P_{r}=P_{t}\left[1-\left(1-R_{i}\right)^{V_{\mathrm{ij}}}\right](1)$

where $V_{i j}$ represents the number of visits by pollinator $i$ to flower $j$, which is a subset of $V_{i}$, the total number of visits made by the pollinator in the plant community. Assuming that the visits made by the pollinator $i$, $V_{i}$, are equally distributed among all the flowers visited by the pollinator, the number of visits to the focal flower by pollinator $i$ is

$V_{\mathrm{ij}}=\frac{V_{i}}{A_{a}+\sum A_{i}}(2)$

where $A_{a}$ represents the abundance of the focal species and $\Sigma A_{\mathrm{i}}$ represents the total abundance of flowers for each plant species visited by the pollinator $i$ excluding the focal species. In this model I treat all plant species as being equally attractive to each pollinator (variable attractiveness could be considered by weighting $A_{a}$ by the relative attractiveness of the focal species).

Considering the role of floral abundance and competition for a limited number of pollinator visits, the number of pollen grains removed from a focal flower by a given pollinator can be expressed as

$P_{r}=P_{t}\left[1-\left(1-R_{i}\right)^{\frac{V_{i}}{A_{a}+\Sigma A_{i}}}\right](3)$

The proportion of pollen grains removed by the pollinator $i$ that reach a conspecific stigma depends on the pollen carryover capacity of the pollinator, $C_{i}$ - the proportion of pollen carried over to each subsequent visit. Pollen carryover only accounts for the proportion of pollen that is still in circulation for potential pollination. Pollen groomed and accumulated on the scopae or corbiculae of bees is therefore not considered in pollen carryover as this pollen very rarely contributes to pollination (Thorp 2000). For a given individual of the pollinator $i$, as with each new visit the amount of pollen remaining on the pollinator body declines at a rate $C_{i}$, the proportion of grains remaining on the individual pollinator follows an exponential decay (Lertzman \& Gass 1983; Campbell 1985; Robertson 1992) (although longer or shorter than exponential tails have been observed; Morris et al. , 1994; Holmquist et al. , 2012). From the pollen grains deposited on the individual pollinator body, as pollen is lost with each visit, the amount that will reach a conspecific stigma is a function of the number of interspecific visits made by the pollinator before reaching a conspecific flower, which is a function of the reciprocal of the proportional floral abundance of the focal species in the community of flowers visited by pollinator $i:\left(A_{a}+\Sigma A_{i}\right) / A_{a}$. Therefore, assuming that the pollinator does not exhibit floral constancy (floral constancy is considered in Appendix S1 by weighting $\Sigma A_{i}$ by the reciprocal of the degree of floral constancy exhibited by the pollinator), the proportion of the removed pollen grains by individuals of pollinator " $i$ " that reach conspecific stigmas is equivalent to

$$
C_{i} \frac{A_{a}+\sum A_{i}}{A_{a}}
$$


Floral constancy can be considered as temporal specialization (Waser 1986; Amaya-Márque 2009), and therefore has a similar impact on pollen transport as fixed specialization (not behaviourally flexible), which is represented in the model by $A_{a}+\Sigma A_{i}$. Considering both the amount of pollen removed by the pollinator $i$ and the proportion of this pollen that is deposited on conspecific stigmas, the total number of pollen grains deposited on conspecific stigmas, $P_{d}$, is expressed as

$P_{d}=P_{t}\left[1-\left(1-R_{i}\right)^{\frac{V_{i}}{A_{a}+\sum A_{i}}}\right] \bullet C_{i} \frac{A_{a}+\sum A_{i}}{A_{a}}(4)$

Equation (4) determines the effect of specialization on a given pollinator on the pollination success of the focal plant species. The effect of specialization on different pollinators can be evaluated by comparing the value of $P_{d}$ for exclusive pollination by different pollinators.

When multiple pollinators visit the focal plant species (generalization on a subset of the available pollinators), considering that the number of pollen grains removed from a flower diminishes with each new visit, the number of pollen grains removed by a given pollinator $i$ depends on visitations by other pollinators. Pollinator $i$ therefore removes a subset of the total amount of pollen removed by all the pollinators visiting the focal flower. Assuming random visitation order between individuals of the different pollinators, the total proportion of pollen grains removed from the focal flower, $P_{r}$, can be expressed as the product of the proportion of pollen removed by each pollinator alone.

$$
\prod_{i=1}^{n}\left(1-R_{i}\right)^{\frac{V_{i}}{A_{a}+\sum A_{i}}}
$$

From the total number of pollen grains removed from the focal flower, the contribution of pollinator $i$ to the number of removed pollen grains is relative to the proportion of visits to the focal flower made by the pollinator $i$

$$
\frac{\left(\frac{V_{i}}{A_{a}+\sum A_{i}}\right)}{\left(\sum_{i=1}^{n} \frac{V_{\mathrm{ij}}}{A_{a}+\sum A}\right)}
$$

and its pollen removal rate relative to the removal rate of the other pollinators visiting the focal flower.

$$
\frac{R_{i}}{\left(\frac{\sum_{i=1}^{n} R_{i}}{n}\right)}
$$

The number of pollen grains removed by the pollinator $i$ can therefore be expressed as

$$
\left[\prod_{i=1}^{n}\left(1-R_{i}\right)^{\frac{V_{i}}{A_{a}+\sum A_{i}}}\right] \cdot \frac{\left(\frac{V_{i}}{A_{a}+\sum A_{i}}\right)}{\left(\sum_{i=1}^{n} \frac{V_{\mathrm{ij}}}{A_{a}+\sum A}\right)} \cdot \frac{R_{i}}{\left(\frac{\sum_{i=1}^{n} R_{i}}{n}\right)}
$$

The proportion of the removed pollen grains that reaches conspecific stigmas is measured in the same way as for equation (4). The individual contribution of each pollinator to $P_{d}$ is summed and the total amount of pollen deposited on conspecific stigmas corresponds to

$$
P_{t}=\sum_{i=1}^{n} P_{t}\left\{\left[1-\prod_{i=1}^{n}\left(1-R_{i}\right)^{\frac{V_{i}}{A_{a}+\sum A_{i}}}\right] \bullet \frac{\left(\frac{V_{i}}{A_{a}+\sum A_{i}}\right)}{\left(\sum_{i=1}^{n} \frac{V_{\mathrm{ij}}}{A_{a}+\sum A}\right)} \bullet \frac{R_{i}}{\left(\frac{\sum_{i=1}^{n} R_{i}}{n}\right)}\right\} \bullet C_{i} \frac{A_{a}+\sum A_{i}}{A_{a}}
$$


Equation (5) is a generalization of equation (4) and determines the effect of pollination by a combination of pollinators on the pollination success of the focal plant species.

\section{Adaptive foraging}

Many pollinators can adjust their foraging behavior in response to resource availability (i.e. adaptive foraging) (Goulson 1999). When considering adaptive foraging, a pollinator's foraging preference for a given floral host is related to the reward intake from that host relative to the average reward intake from all its plant hosts (Valdovinos et al. 2016). In other words, a plant with higher reward content on average will be more attractive to its pollinators than a plant with lower reward content. In the model, reward availability (and therefore reward intake) is determined by the number of pollinator visits per flower, with more visits resulting in greater reward depletion. When floral rewards are produced at a constant rate, or are produced only once at the time of flower opening, the average quantity of reward available per flower of the plant species $a, Q_{a}$, is directly linked to the average number of pollinator visits received per flower. $Q_{a}$ can therefore be expressed as a proportion of the maximal reward content, corresponding to

$$
Q_{a}=\frac{1}{1+\frac{\mathrm{Vr}_{j}}{F_{a}}}
$$

where $V r_{j}$ corresponds to the per flower pollinator visitation rate to plant a measured in the same unit as the reward production rate, $F_{a} . F_{a}$ represents the product of the flower production rate and reward replenishment rate. The denominator "1" corresponds to the initial reward content of a flower, such that previously unvisited flowers contain the maximal amount of reward. In many plant species, however, reward (mostly nectar) is replenished dynamically following pollinator visits (Castellanos et al. 2002; Juan \& Ornelas 2004; Bobrowiec \& Oliveira 2012; Ogilvie et al.2014; Ye et al. 2017). With dynamic replenishment, the reward replenishment rate is initially high following reward removal, but eventually plateau. When considering dynamic reward replenishment, $Q_{a}$ corresponds to

$$
Q_{a}=1-\left(1-F_{a}\right)^{\mathrm{Vr}_{j}}
$$

where $F_{a}$ is the initial replenishment rate after a pollinator visit, expressed as a proportion of the maximal reward content. Assuming that reward production is equal among plant species, the total number of visits to plant $a$ by pollinator $i$ is

$$
\mathrm{Vt}_{\mathrm{ij}}=V_{\mathrm{i}} \bullet \frac{A_{a} \bullet Q_{a}}{\sum_{a=1}^{n} A \bullet Q}
$$

and the number of visits to the focal flower by pollinator $i, V_{i j}$ is equal to $V t_{i j} / A_{a}$.

\section{Methods}

\section{Importance of pollinator quality and quantity}

To determine the effect of floral abundance on the relative importance of the quantity and quality components of pollination for pollination success, using equation (4), I compared the impact of variations in those components on conspecific pollen receipt at different floral abundances. Equation (4) offers an explicit definition of which parameters constitute the quantity and quality components of pollination. Factors affecting the quantity of pollen removed (the left part of the equation) - pollinator abundance and pollen removal rate - are defined as the quantity component. Factors affecting pollen transport efficiency (the right part of the equation) - pollinator carryover capacity and specialization - are defined as the quality component. Given that removal rate does not affect pollen transport efficiency (e.g. a pollen forager will be of low quality despite having a high removal rate), removal is only considering to affect pollination quantity. I used $\Sigma A_{i}$ 
, the total floral abundance of all the plant species visited by the pollinator (see equation 4) as a proxy for pollinator specialization. The number of pollen grains produced by the focal flower deposited on a conspecific stigma (equation 4) was compared at low and high values of the parameters (Table 1) while maintaining the other parameters constant. The proportional change (high - low ) / high produced an estimate of the importance of variation of those parameters on pollination success. The importance of the different parameters for pollination success was compared for a range of floral abundances from 2 to 1500 (at least two flowers are required for cross-pollination).

Low, medium and high values of pollen carryover and pollen removal were parameterized based on data from the literature (Table 1). From a literature survey of 18 studies on plant species and pollen vectors, Robertson (1992) reported a range in pollen carryover from $50.2 \%$ to $94.7 \%$. I used values of $0.55,0.73$ and 0.9 as low, medium and high values of pollen carryover in the model respectively. The values of pollen removal were selected following Thomson (2003) who modeled pollen delivery as a function of low and high values of pollen removal of 0.3 and 0.7 respectively. In the model, I used values of $0.3,0.5$ and 0.7 as low, medium and high values of pollen removal respectively. These values encompass the pollen removal values measured in various systems (e.g. Wolfe and Barrett 1989, Young and Stanton 1990, Harder 1990, Thostesen and Olesen 1996). Low and high values of total number of visits by the pollinator in the community and abundance of the flower species were not based on empirical data, but were rather selected such that, (1) for plant species of intermediate abundance, most pollen grains were removed $(>90 \%)$ at high values of pollinator visits and low abundance of other flower species, while $(2)$ a minority of grains $(>50 \%)$ were removed at low pollinator visits and high abundance of other plant species. These scenarios reflect low and high pollinator limitation respectively and should therefore encompass most real-life situations. Medium values of these parameters were determined as the mean between low and high values (high and low values of total number of visits by the pollinator in the community and abundance of the other flower species represent a two-fold increase and decrease from the medium values respectively).

Because the number of pollen grains deposited on conspecific stigmas might be sensitive to the choice of values of the parameters used for the mathematical model, I compared the impact of variations in pollen carryover, pollen removal, pollinator visitation and specialization on conspecific pollen receipt at each possible set of values of the other parameters (low, medium, and high). I used these alternative parameter values to set upper and lower bounds for the estimated importance of the quantity and quality components of pollination. Intermediate values of the importance of a parameter on pollination success correspond to the values obtained while all other parameters were set to medium values. Upper and lower values correspond to the maximal and minimal values obtained among all alternative values of the other parameters respectively. Essentially, the upper and lower values of the estimated importance of the quality and quantity components of pollination indicate the degree to which the estimate varies as a function of variation in the different parameters of the mathematical model and serve as a confidence interval.

\section{Plant-pollinator network simulations}

Using equation (5), I verified how variation in floral abundance affects the structure of plant-pollinator networks. Each simulated network was composed of a community of 10 pollinators and 12 plant species. Pollinator communities were assembled by randomly sampling values of pollen carryover and removal for each pollinator from uniform distributions using the runif function in $\mathrm{R}$ ( $\mathrm{R}$ core team, 2020) with maximal and minimal values of 0.9 and 0.55 , and 0.7 and 0.3 for carryover and removal respectively. The number of visits made by the different pollinators (relative to their abundance) was sampled from a Poisson lognormal distribution using the rpoilog function in the $\mathrm{R}$ package sads (Prado et al. 2018). Poisson log-normal distributions are often used to characterize community species-abundance distributions (Baldridge et al. 2016).

After randomizing plant species order, each plant species colonized the pollinator community successively until all species had colonized the community. For each colonization event, the plant species could evolve to be pollinated by any possible combination of pollinators in the community. The combination resulting 
in the highest pollination success was selected as the evolutionary outcome for the plant species (assuming no restriction on the evolution of different pollination systems). Considering that new colonization events affect competition and interspecific pollen transfer, after all plant species colonized the community, each plant species could continue evolving different pollination systems. This was simulated by allowing for five successive times each species in random order to evolve a new pollination system. This assured that the networks had the opportunity to reach a stable evolutionary solution.

Plant pollination success associated with the evolution of pollination by the different possible combinations of pollinators was calculated and compared by inputting the simulated parameters (see Table 2) in equation (5). The model used for the simulations incorporated adaptive foraging by using equation (6) and (8) to characterize reward availability and pollinator visitation. The reward production rate, $F_{a}$, was set to 1 such that $V r_{j}=V_{j}$. Equation (6) therefore directly corresponded to

$$
Q_{a}=\frac{1}{1+V_{j}}
$$

such that reward quantity was directly related to the number of pollinator visits received. Adaptive foraging was updated dynamically with every change in interaction and affected pollination system evolution. Competition for visits by the different pollinators was dynamically updated with each new colonization event.

Sets of 100 simulations were run for plant communities of either variable interspecific floral abundance or same floral abundance at low (average of 100 flowers), intermediate low (average of 250 flowers), intermediate high (average of 500 flowers) and high floral abundance (average of 1000 flowers). These floral abundance values were selected to encompass a range of situations from the removal of most pollen grains produced by flowers $(>99 \%)$ to the removal of a low portion of pollen grains $(<60 \%)$ and was given by

$$
R^{\frac{\sum V}{n}}
$$

For the simulations with variable floral abundance, plant communities were assembled by randomly sampling plant abundances for each species from a Poisson log-normal distribution.

To analyse the properties of the simulated plant-pollinator networks, I measured network nestedness, connectance and average number of shared pollinators per plant species (measure of niche overlap) using the 'networklevel' function in the R package bipartite (Dormann et al. 2020). 'Networklevel' produces values of nestedness in degrees (T). Following Bascompte et al. (2003) nestedness, N, was defined as $\mathrm{N}=(100-$ $\mathrm{T}) / 100$ with values ranging from 0 to 1 (where 1 represents maximum nestedness). Pollinators in this model were treated as functional groups of pollinator species with similar attributes which prevented the formation of modules of pollinators sharing similar attributes. Network analysis therefore did not include measures of modularity. Variability in degree of specialization within communities was measured as the standard deviation in the number of pollinators visiting the different plant species constituting the plant community of a simulated network.

I ran sets of simulations in which pollinator abundance was either (1) constant and independent of floral resource abundance, or (2) variable and proportional to the resources available to each pollinator. In plantpollinator systems exhibiting very tight mutualisms where pollinators depends on their plant host throughout the entirety of their life cycle (e.g. figs and fig-wasps, yucca and yucca-moths), pollinator abundance may be tightly linked to its floral host abundance. In most plant-pollinator systems, however, pollinator abundance is weakly linked to floral host abundance since pollinator populations are limited by other factors such as nest sites, larval host availability or territory (Burd 1995; Pauw 2007, 2013; Benadi \& Pauw 2018). For this reason, and because the models with and without variation in pollinator abundance produced qualitatively similar results, I present the results from the simulations without variation in pollinator abundance in this paper (results and details on the methodology for simulations with variation in pollinator abundance can be found in the supplementary material; Appendix S1). 
I verified the robustness of the conclusions drawn from the model to variation in parameter values beyond the ones used for the standard model and simulations. Additionally, I verified the robustness of the model to the presence of dynamic reward replenishment (equation 7) and the absence of adaptive foraging. Finally, although the model presented here does not incorporate flower consistency, I ran supplementary sets of simulations with different degrees of flower constancy. Given that the general conclusions of the study were robust to those alternative models and parameter values, detailed results from the alternative sets of simulations are presented as supplementary information (Appendix S1).

\section{Pollination system evolution as a function of floral abundance}

Using the simulated plant-pollinator networks of the variable floral abundance plant communities with intermediate high average abundances, I tested how floral abundance affects the degree of floral specialization and whether different floral abundances lead to adaptation to different pollinators. For each simulated plantpollinator network, after all plant species had colonized the community, a new plant colonist invaded the community. I varied the new colonist's abundance and recorded the subset of pollinators on which the plant evolved at each abundance value.

Diversification of pollination systems as a function of variation in floral abundance, community context and pollinator assemblage

I then tested if changes in species abundance associated with the colonization of new communities, as well as inter-community variation in plant species composition and pollinator assemblage can drive pollination system diversification in plant clades. Each simulation consisted of a new colonist successively invading and adapting to 20 different plant communities, representing the diversification of a plant clade extending its geographical range. Assuming that 1) there is some degree of variation in the composition of the different communities and 2) the composition of each community is likely to be most similar to the community nearest to it in space, the attributes of each new colonized community (abundance of the new colonist, abundance of the other plant species, or of the different pollinators) were randomly sampled from a normal distribution characterized by a mean equal to the average value of the attributes of the last colonized community. Different sets of 100 simulations were run with different degrees of variation of the normal distribution from a coefficient of variation of 0.01 to 1 , with increments of 0.01 between sets of simulations (the coefficient of variation corresponds to the standard deviation standardized to represent a proportion of variation around the average). Distributions were truncated at minimum values of 2 (assuming that at least two individuals are necessary for species survival) to avoid sampling negative values using the 'rtruncnorm' function from the R package 'truncnorm' (Mersmann et al. 2018). The initial plant-pollinator networks used for the simulations were generated in the same way as in the previous simulations.

Three different sets of simulations were performed in which the invaded communities varied in either 1) the abundance of the new colonist, 2) the abundance of the existing plant species in the community and 3) the abundance of the different pollinator species. The subset of pollinators on which the colonist evolved toward were recorded for each simulation.

\section{Results}

The impact of pollinator abundance and pollen removal rate on plant pollination success increased with floral abundance, while the impact of pollen carryover and pollinator specialization decreased with floral abundance (fig. 1A-D). Overall, the quality component of pollination was more important for pollination success than the quantity component at low floral abundance, while the quantity component was more important for pollination success at high abundance (fig. 1E).

For simulated plant communities in which plant species varied in abundance, the different species exhibited variable degrees of specialization within the communities (average number of links \pm standard deviation $=2.17 \pm 0.29$ ) (fig. 2A, B). In contrast, in simulated plant communities where all plant species were of low abundance, most species specialized on a limited subset of the available pollinators (average number of 
links \pm standard deviation $=1.58 \pm 0.32$ ) (Fig. 2C, D). In plant communities composed of abundant plant species, most species generalized on a high proportion of the pollinators available (average number of links \pm standard deviation $=3.41 \pm 0.48)($ Fig. $2 \mathrm{E}, \mathrm{F})$.

The simulated plant communities in which species varied in abundance exhibited more variation in degree of specialization among species relative to the communities with no variation in plant abundance (Fig. 3A). This effect was more pronounced in communities with high average floral abundance. The communities with variation in abundance shared fewer pollinators between plant species (Fig. 3B) and produced more nested (Fig. 3C) and less connected plant-pollinator networks (Fig. 3D). Incorporating adaptive foraging produced less connected networks while high flower constancy of the pollinators had the opposite effect (Appendix S1).

The subset of available pollinators to which a new plant colonist evolved was a function of its floral abundance. At very low floral abundance, the colonist specialized on pollinators weakly exploited by the other plant species, thereby reducing competition via interspecific pollen transfer (Fig. 4). These less-preferred pollinators were often relatively rare and had a low carryover capacity, as pollinators with high abundance and carryover were generally exploited by several plant species. From very low to relatively low abundance, there was a tendency for the new colonist to shift toward specialization on a subset of pollinators with high carryover rather than low competition. This pattern was less pronounced when all plant species were of low abundance and all pollinators were therefore weakly exploited (at average flower abundance of 100, see Appendix S1). In this case, given that competition was low for all pollinators, high carryover was favored at both very low and low abundance. From intermediate to high abundance, the new colonist favoured more abundant pollinators. Generalization increased with abundance, but only at very high abundance were the majority of the available pollinators exploited. Pollinator competition (degree of specialization of the pollinator) and carryover were opposed such that pollinators with high carryover capacity were exploited by several plant species, while pollinators with low carryover capacity were less exploited. This pattern resulted in plants evolving on pollinators with less competition in average at high plant abundance relative to low abundance. Indeed, increased generalization at high abundance resulted in the incorporation of both pollinators with high carryover and low competition (specialized pollinators) comparatively to only pollinators with high carryover (and therefore highly exploited pollinators) at low abundance.

For the simulated plant clades colonizing new communities, diversity in pollination systems increased with variation in species abundance within the clade, as well as with variation in plant community composition and pollinator assemblage (Fig. 5).

\section{Discussion}

Many plant lineages and communities are characterized by high floral diversity (Van der Niet \& Johnson 2012). However, the causes of floral diversification and specialization remain elusive (Kay \& Sargent 2009; Johnson 2010; Van der Niet \& Johnson 2012; Armbruster 2017). Here I propose that a species' relative abundance in a community determines the pollination system offering the optimal evolutionary solution (Fig. 1, 4). Given that abundance is evolutionarily and ecologically labile (Ricklefs 2010; Loza et al. 2017), shifts in abundance associated with the colonization of new habitats or geographic ranges could promote floral diversification. This model complements the Grant-Stebbins model in which flower diversification results from geographical variation in pollinator assemblages (Grant \& Grant 1965; Stebbins 1970). In this more holistic view, floral diversification is the result of variability in pollinator assemblages (Fig. 5c), floral abundance (Fig. 5a), and plant-community composition (Fig. 5b). This perception considerably relaxes the conditions under which floral diversification occurs and offers an explanation for the variability in pollinator use and degree of specialization within and among communities.

Within communities, the presence of interspecific variation in species abundance increased diversity in degree of generalization and decreased niche overlap in pollinator use (Fig. 3). Such variability also contributed to producing more realistic network structures. Indeed, while networks from communities without variation 
in abundance were frequently less nested and more connected then empirical plant-pollinator networks, communities with variable species abundance produced networks with nestedness and connectance within the range of empirical values (Fig. 3, compared to values of nestedness of 0.59 to 0.98 from 25 plant-pollinator networks in Bascompte et al. 2003 and values of connectance of 0.02 to 0.29 from 29 networks in Olesen \& Jordano 2002).

In the simulated plant communities, flower specialization was observed at low abundance while generalization was favoured at higher abundance (Fig. 2A, B, Fig. 4), a pattern consistent with the frequently observed link between abundance and degree of generalization in plant-pollinator networks (Jordano et al. 2002; Bascompte et al. 2003; Vázquez \& Aizen 2003). While the cause of this pattern is debated (Dorado et al. 2011; Fort et al. 2016), the model presented here suggests that the link between abundance and generalization can originate from a selective advantage of generalization at high abundance. Furthermore, simulated plant communities composed of plant species of low abundance resulted in widespread specialization, while communities of high abundance species were associated with generalized pollination (Fig. 2). This observation is consistent with the widespread floral specialization characterizing highly diverse plant communities composed mostly of rare species, such as in Mediterranean and tropical climates (Johnson \& Steiner 2000; Vamosi et al. 2006). In such communities, plants should be under stronger selective pressure for specialization in order to avoid pollen loss from inefficient carryover and interspecific pollen transfer (Feinsinger 1983, Johnson and Steiner 2000).

Interestingly, while at moderately low abundance plants specialized on a subset of pollinators with high carryover capacity, at very low abundance the species frequently specialized on pollinators of low abundance and carryover (Fig. 4). Those pollinators were less exploited by more abundant plant species, which instead evolved pollination by abundant and efficient carriers of their pollen, offering a competition-free space for very rare species. This pattern of plant community assembly can be explained by the increasing probability of interspecific visits with increasing plant rarity, exacerbating the importance of interspecific pollen loss at very low abundance. The propensity for rare plant species to fill up unexploited pollination niches has the potential to give rise to the evolution of unique pollination systems, potentially contributing to the impressive diversity in modes of pollination characterizing tropical and Mediterranean communities.

The mathematical model and the simulated plant-pollinator networks demonstrate that, from low to intermediate abundance, plants should specialize on a subset of pollinators offering the optimal combination of pollination quantity and quality components (Fig. 1, 4). However, as plant abundance increases and most pollinators are not sufficiently abundant to remove the majority of pollen grains, highly generalized pollination should be favoured. But what happens at the extreme end of the plant abundance spectrum, when the entire pollinator community cannot provide enough visits to prevent pollen limitation? In those conditions, perhaps the best strategy is for plants to relax their dependence on biotic pollen vectors. While the evolution of wind pollination from animal pollination has mostly been attributed to reduced reliability of animal pollinators, most wind-pollinated plants are characterized by large population size and high density (Culley et al. 2002; Friedman \& Barrett 2009). Indeed, it seems doubtful that any combination of pollinators could adequately pollinate the thousands of flowers per square metre characterizing the bloom of temperate deciduous trees or Poaceae grasslands. Moreover, despite wind representing a relatively inefficient system of pollen transport, the high abundance characterizing most wind-pollinated plants reduces the importance of pollen vector efficiency.

Several theoretical models emphasize the importance of fitness trade-offs in the evolution of flower specialization (Aigner 2001; Sargent \& Otto 2006; Muchhala 2007). Such trade-offs are expected to occur when adaptation to a pollinator decreases the effectiveness of pollination by other pollinators. However, despite having been detected in some studies, fitness trade-offs in the effective use of different pollinators are often weak or absent (see Armbruster 2014, 2017). Hence, it seems unlikely that floral specialization is the sole result of trade-offs. Here, similar to Muchhala et al. (2010), who investigated the role of interspecific pollen transfer in flower specialization, I demonstrate that specialization can evolve without trade-offs. Rather, specialization should be advantageous when pollinator quantity is less limiting than pollinator quality.

When present, fitness trade-offs in the effective use of different pollinators should increase floral specialization. 
However, the model presented here is consistent with the perception that floral specialization might be governed not only by adaptation to increase pollen removal and deposition by the most effective pollinator, but also by the exclusion of less efficient ones (Thomson 2003; Castellanoset al. 2004; Muchhala et al. 2010; Armbruster 2017). Paralleling evolution toward the most effective pollinator, exclusion of less efficient pollinators through the evolution of pollinator filters could produce trade-offs if it also excludes efficient but infrequent pollinators. In other words, pollinator filters might rarely allow singling out unwanted pollinators. For instance, the evolution of long nectar spurs prevents access to pollinators with short mouthparts, even if some of those pollinators might act as occasional but efficient visitors. Plants might therefore often evolve a high degree of evolutionary specialization despite several visitors acting as effective pollinators due to the limited capacity to maintain pollination by a subset of effective pollinators while precisely excluding the ineffective ones.

\section{Caveats}

In the model, in order to demonstrate the role of floral abundance in determining the optimal pollination system, plants could evolve on any combination of pollinators without evolutionary restrictions. Plants, however, have constrained abilities to track the most effective pollination system (Fenster et al. 2004). Evolution occurs along the lines of least resistance contingent on the genetic material available, making some shifts in pollination systems more likely to occur in certain plant lineages (Stebbins 1970; Van der Niet \& Johnson 2012). Those evolutionary limitations represent an important nuance in flower diversification. Plants should adapt to the most effective pollination strategy that represent a line of least resistance. Therefore, many plants are expected to not be optimally adapted to their pollination environment. For instance, bilateral symmetry is considered to facilitate floral specialization (Sargent 2004) and radially symmetrical flowers may have limited ability to evolve floral specialization. Additionally, the evolution of specialized pollination rewards, such as oil and fragrance, that allow pollination by specific pollinator functional groups (e.g. oil and fragrance collecting bees) might often be contingent on particular floral traits (preaptations) already being in place (Armbruster 2011). Although transitions between "normal" and specialized rewards and between different specialized rewards are frequent (Armbruster 2011), shifts in pollination systems that require transitions between rewards might be more evolutionary constrained than shifts between pollinators using the same reward.

Even in the absence of evolutionary constraints, because there is a net flux of genes from large to small populations, the smallest, and often peripheral, populations of a given plant species are expected to match the local selective pressures relatively poorly (García-Ramos \& Kirkpatrick 1997; Kirkpatrick \& Barton 1997; Kay \& Sargent 2009). Considering the importance of floral abundance in determining the optimal pollination strategy, small peripheral populations should rarely match such optimal conditions. Furthermore, large fluctuations in population abundance might prevent adaptation to the most effective pollinator when such fluctuations happen fast enough that adaptive tracking is not possible.

For a given plant species, its flower abundance in a community is the result of the species abundance and the average number of flowers produced per individual. Although I focused on variation in species abundance in the model, variation in average number of flowers per individual plant should have an additional direct effect on fitness: all else being equal, an individual that produces more flowers will also produce more gametes (pollen and ovules). Hence, in the instances where adapting to more effective pollinators is costly, flower evolution might conflict with the direct advantage of producing more flowers.

\section{Conclusion and future directions}

In virtually all communities, species vary widely in their abundance. In this article, I demonstrate that this variability could explain a part of the angiosperms' remarkable floral diversity. The model presented here offers a potential framework to understand the evolution toward different pollination systems. According to this model, the optimal pollination system is a function of the pollinator assemblage (which varies geographically according to the Grant-Stebbins model), the plant community in which a species is embedded, and its floral abundance. This more holistic view integrating the plant community context with selection exerted 
by different pollinators promises to improve our understanding of the ecological processes involved in flower diversification.

Future studies should investigate how this mechanism operates in natural systems. In support of the model, some studies have shown that the strength of selection on floral traits can be affected by floral density (see Eisen et al. 2020 and references therein). An important next step would be to investigate how floral abundance affects the strength and direction of selection exerted by different pollinators. Such studies should be performed at the scale of whole populations to encompass representative variation in the pollination processes affected by floral abundance. Studies investigating how different pollination systems are associated with distinct floral abundances could also be very informative, although such studies would require careful consideration of historical contingency and spatiotemporal variation in species' abundances.

\section{ACKNOWLEDGMENTS}

I thank Jessica Forrest, Colleen Smith, Lydia Wong and the members of the Forrest laboratory for valuable comments on the manuscript. Financial support was provided through a doctoral scholarship from the Natural Sciences and Engineering Research Council of Canada.

\section{REFERENCES}

Aigner, P.A. (2001). Optimality modeling and fitness trade-offs: when should plants become pollinator specialists? Oikos , 95, 177-184.

Amaya-Márque, M. (2009). Floral constancy in bees: a revision of theories and a comparison with other pollinators. Rev. Colomb. Entomol. , 35, 206-216.

Armbruster, S.W. (2011). Evolution and ecological implications of "specialized" pollinator rewards. In: Evolution of plant-pollinator relationships (ed. Patiny, S.). Cambridge University Press, New York., pp. 44-67.

Armbruster, W.S. (2014). Floral specialization and angiosperm diversity: phenotypic divergence, fitness trade-offs and realized pollination accuracy. AoB Plants , 6, plu003.

Armbruster, W.S. (2017). The specialization continuum in pollination systems: diversity of concepts and implications for ecology, evolution and conservation. Funct. Ecol. , 31, 88-100.

Baldridge, E., Harris, D.J., Xiao, X. \& White, E.P. (2016). An extensive comparison of species-abundance distribution models.PeerJ , 2016, e2823.

Bascompte, J., Jordano, P., Melián, C.J. \& Olesen, J.M. (2003). The nested assembly of plant-animal mutualistic networks. Proc. Natl. Acad. Sci. U. S. A., 100, 9383-9387.

Benadi, G. \& Pauw, A. (2018). Frequency dependence of pollinator visitation rates suggests that pollination niches can allow plant species coexistence. J. Ecol. , 106, 1892-1901.

Bergamo, P.J., Susin Streher, N., Traveset, A., Wolowski, M. \& Sazima, M. (2020). Pollination outcomes reveal negative density-dependence coupled with interspecific facilitation among plants. Ecol. Lett. , 23, $129-139$.

Bobrowiec, P.E.D. \& Oliveira, P.E. (2012). Removal effects on nectar production in bat-pollinated flowers of the Brazilian Cerrado.Biotropica , 44, 1-5.

Burd, M. (1995). Pollinator behavioural responses to reward size inLobelia Deckenii : no escape from pollen limitation of seed set.J. Ecol. , 83, 865 .

Campbell, D.R. (1985). Pollen and gene dispersal: the influences of competition for pollination. Evolution. , 39, 418-431.

Caruso, C.M. (2000). Competition for pollination influences selection on floral traits of ipomopsis aggregata. Evolution. , 54, 1546-1557. 
Caruso, C.M. (2002). Influence of plant abundance on pollination and selection on floral traits of ipomopsis aggregata .Ecology , 83, 241-254.

Castellanos, M.C., Wilson, P. \& Thomson, J.D. (2002). Dynamic nectar replenishment in flowers of Penstemon (Scrophulariaceae).Am. J. Bot. , 89, 111-118.

Castellanos, M.C., Wilson, P. \& Thomson, J.D. (2004). "Anti-bee" and "pro-bird" changes during the evolution of hummingbird pollination inPenstemon flowers. J. Evol. Biol. , 17, 876-885.

Culley, T.M., Weller, S.G. \& Sakai, A.K. (2002). The evolution of wind pollination in angiosperms. Trends Ecol. Evol. , 17, 361-369.

Darwin, C. (1877). The various contrivances by which orchids are fertilised by insects . John Murray, London, UK.

Dorado, J., Vazquez, D.P., Stevani, E.L. \& Chacoff, N.P. (2011). Rareness and specialization in plantpollinator networks.Ecology , 92, 19-25.

Dormann, C.F., Fruend, J. \& Gruber, B. (2020). Package bipartite: visualising bipartite networks and calculating some (ecological) indices. https://CRAN.R-project.org/package=bipartite.

Duffy, K.J. \& Stout, J.C. (2008). The effects of plant density and nectar reward on bee visitation to the endangered orchidSpiranthes romanzoffiana . Acta Oecologica , 34, 131-138.

Eisen, K.E., Wruck, A.C. \& Geber, M.A. (2020). Floral density and co-occurring congeners alter patterns of selection in annual plant communities. Evolution., in press.

Essenberg, C.J. (2012). Explaining variation in the effect of floral density on pollinator visitation. Am. Nat. , 180, 153-66.

Fattorini, R. \& Glover, B.J. (2020). Molecular Mechanisms of Pollination Biology. Annu. Rev. Plant Biol. $, 71,487-515$.

Feinsinger, P. (1983). Coevolution and pollination. In:Coevolution (eds. Futuyma, D.J. \& Slatkin, M.). Sinauer Associates, Sunderland, MA, pp. 282-310.

Feinsinger, P., Tiebout, H.M. \& Young, B.E. (1991). Do tropical bird-pollinated plants exhibit densitydependent interactions? Field experiments. Ecology , 72, 1953-1963.

Fenster, C.B., Armbruster, W.S., Wilson, P., Dudash, M.R. \& Thomson, J.D. (2004). Pollination syndromes and floral specialization.Annu. Rev. Ecol. Evol. Syst., 35, 375-403.

Ferreiro, G., Baranzelli, M.C., Sersic, A.N. \& Cocucci, A.A. (2017). Patterns of phenotypic selection for oil and nectar in Monttea aphylla (Plantaginaceae) in a geographic mosaic of interactions with pollinators. Flora , 232, 47-55.

Fort, H., Vazquez, D.P. \& Lan, B.L. (2016). Abundance and generalisation in mutualistic networks: solving the chicken-and-egg dilemma. Ecol. Lett. , 19, 4-11.

Friedman, J. \& Barrett, S.C. (2009). Wind of change: new insights on the ecology and evolution of pollination and mating in wind-pollinated plants. Ann. Bot. , 103, 1515-1527.

Garcia-Ramos, G. \& Kirkpatrick, M. (1997). Genetic models of adaptation and gene flow in peripheral populations. Evolution. , 51, 21-28.

Geber, M.A. \& Moeller, D.A. (2006). Pollinator responses to plant communities and implications for reproductive character evolution. In:Ecology and evolution of flowers (eds. Harder, L.D. \& Barrett, S.C.H.). Oxford University Press, New York, NY, pp. 102-119.

Ghazoul, J. (2005). Pollen and seed dispersal among dispersed plants.Biol. Rev. , 80, 413-443. 
Gomez, J.M. \& Regino, Z. (2006). Ecological factors that promote the evolution of generalization in pollination systems. In:Plant-pollinator interactions: from specialization to generalization (eds. Waser, N.M. \& Ollerton, J.). University of Chicago University Press, Chicago, IL, pp. 145-166.

Goulson, D. (1999). Foraging strategies of insects for gathering nectar and pollen, and implications for plant ecology and evolution.Perspect. Plant Ecol. Evol. Syst. , 2, 185-209.

Grant, V. \& Grant, K. (1965). Flower pollination in the Phlox family . Columbia University Press, New York, NY.

Harder, L.D. (1990). Pollen removal by bumble bees and its implications for pollen dispersal. Ecology , 71, $1110-1125$.

Harder, L.D. \& Johnson, S.D. (2009). Darwin's beautiful contrivances: evolutionary and functional evidence for floral adaptation. New Phytol. , 183, 530-545.

Hegland, S.J. (2014). Floral neighbourhood effects on pollination success in red clover are scale-dependent. Funct. Ecol. , 28, 561-568.

Hernandez-Hernandez, T. \& Wiens, J.J. (2020). Why are there so many flowering plants? A multi-scale analysis of plant diversification.Am. Nat. , 195, 000-000.

Herrera, C.M., Castellanos, C. \& Nica Medrano, M. (2006). Geographical context of floral evolution: towards an improved research programme in floral diversification. In: Ecology and Evolution of Flowers(eds. Harder, L.D. \& Barrett, S.C.H.). Oxford University Press, New York, NY, pp. 278-294.

Holmquist, K.G., Mitchell, R.J. \& Karron, J.D. (2012). Influence of pollinator grooming on pollen-mediated gene dispersal in Mimulus ringens (Phrymaceae). Plant Species Biol. , 27, 77-85.

Johnson, S. (2006). Pollinator-driven speciation in plants. In:Ecology and Evolution of Flowers (eds. Harder, L.D. \& Barrett, S.C.H.). Oxford University Press, New York, NY, pp. 295-306.

Johnson, S.D. (2010). The pollination niche and its role in the diversification and maintenance of the southern African flora.Philos. Trans. R. Soc. B Biol. Sci., 365, 499-516.

Johnson, S.D. \& Steiner, K.E. (2000). Generalization versus specialization in plant pollination systems. Trends Ecol. Evol. , 15, 140-143.

Jordano, P., Bascompte, J. \& Olesen, J.M. (2002). Invariant properties in coevolutionary networks of plantanimal interactions. Ecol. Lett. , 6, 69-81.

Juan, M.O. \& Ornelas, F. (2004). PLANT ANIMAL INTERACTIONS Generous-like flowers: nectar production in two epiphytic bromeliads and a meta-analysis of removal effects. Oecologia , 140, 495-505.

Kay, K.M. \& Sargent, R.D. (2009). The role of animal pollination in plant speciation: integrating ecology, geography, and genetics.Annu. Rev. Ecol. Evol. Syst. , 40, 637-656.

Kirkpatrick, M. \& Barton, N.H. (1997). Evolution of a species' range.Am. Nat. , 150, 1-23.

Koski, M.H. (2020). The role of sensory drive in floral evolution.New Phytol. , 227, 1012-1024.

Lertzman, K. \& Gass, C. (1983). Alternative models of pollen transfer. In: Handbook of experimental pollination biology . Scientific and Academic Editions, New York, NY, pp. 474-489.

Loza, M.I., Jimenez, I., Jorgensen, P.M., Arellano, G., Macia, M.J., Torrez, V.W., et al. (2017). Phylogenetic patterns of rarity in a regional species pool of tropical woody plants. Glob. Ecol. Biogeogr. , 26, 1043-1054.

Mersmann, O., Trautmann, H., Steuer, D. \& Bornkamp, B. (2018). truncnorm: truncated normal distribution. https://CRAN.R-project.org/package=truncnorm. 
Minnaar, C., Anderson, B., L de Jager, M. \& Karron, J.D. (2019). Plant-pollinator interactions along the pathway to paternity.Ann. Bot. , 123, 225-245.

Mitchell, R.J., Flanagan, R.J., Brown, B.J., Waser, N.M. \& Karron, J.D. (2009). New frontiers in competition for pollination. Ann. Bot. , 103, 1403-1413.

Morales, C.L. \& Traveset, A. (2008). Interspecific pollen transfer: magnitude, prevalence and consequences for plant fitness. CRC. Crit. Rev. Plant Sci. , 27, 221-238.

Moreira-Hernandez, J.I. \& Muchhala, N. (2019). Importance of pollinator-mediated interspecific pollen transfer for angiosperm evolution. Annu. Rev. Ecol. Evol. Syst. , 50, 191-217.

Morris, W.F., Price, M. V., Waser, N.M., Thomson, J.D., Thomson, B. \& Stratton, D.A. (1994). Systematic increase in pollen carryover and its consequences for geitonogamy in plant populations. Oikos , 71, 431.

Moyroud, E. \& Glover, B.J. (2017). The evolution of diverse floral morphologies. Curr. Biol. , 27, R941R951.

Muchhala, N. (2007). Adaptive trade-off in floral morphology mediates specialization for flowers pollinated by bats and hummingbirds. Am. Nat. , 169, 494-504.

Muchhala, N., Brown, Z., Armbruster, W.S. \& Potts, M.D. (2010). Competition drives specialization in pollination systems through costs to male fitness. Am. Nat. , 176, 732-43.

Van der Niet, T. \& Johnson, S.D. (2012). Phylogenetic evidence for pollinator-driven diversification of angiosperms. Trends Ecol. Evol. , 27, 353-361.

Van Der Niet, T., Pirie, M.D., Shuttleworth, A., Johnson, S.D. \& Midgley, J.J. (2014). Do pollinator distributions underlie the evolution of pollination ecotypes in the Cape shrub Erica plukenetii ?Ann. Bot. , $113,301-315$.

Ogilvie, J.E., Thomson, J.D., Luo, E.Y. \& Ogilvie, J.E. (2014). Stimulation of flower nectar replenishment by removal: a survey of eleven animal-pollinated plant species. Artic. J. Pollinat. Ecol. , 12, 52-62.

Olesen, J.M. \& Jordano, P. (2002). Geographic patterns in plant-pollinator mutualistic networks. Ecology, 83, 2416-2424.

Palmer, T.M., Stanton, M.L. \& Young, T.P. (2003). Competition and coexistence: exploring mechanisms that restrict and maintain diversity within mutualist guilds. Am. Nat. , 162, S63-S79.

Pauw, A. (2007). Collapse of a pollination web in small conservation areas. Ecology , 88, 1759-1769.

Pauw, A. (2013). Can pollination niches facilitate plant coexistence? Trends Ecol. Evol. , 28, 30-37.

Prado, P.I., Miranda, D. \& Maintainer, A.C. (2018). sads: maximum likelihood models for species abundance distributions. $\mathrm{R}$ package version 0.4.2. https://cran.r-project.org/package=sads.

R core team (2020). R: a language and environment for statistical computing. R Foundation for Statistical Computing, Vienna, Austria. URL https://www.R-project.org/.

Rathcke, B. (1983). Competition and facilitation among plants for pollination. In: Pollination Biology (ed. Real, L.). Academic Press, New York, pp. 305-329.

Ricklefs, R.E. (2010). Evolutionary diversification, coevolution between populations and their antagonists, and the filling of niche space.Proc. Natl. Acad. Sci. U. S. A., 107, 1265-1272.

Robertson, A.W. (1992). The relationship between floral display size, pollen carryover and geitonogamy in Myosotis colensoi (Kirk) Macbride (Boraginaceae). Biol. J. Linn. Soc. , 46, 333-349.

Robertson, A.W. \& Lloyd, D.G. (1993). Rates of pollen deposition and removal in Myosotis colonsoi . Funct. Ecol. , 7, 549 . 
Runquist, R.B. \& Stanton, M.L. (2013). Asymmetric and frequency-dependent pollinator-mediated interactions may influence competitive displacement in two vernal pool plants. Ecol. Lett. , 16, 183-190.

Sargent, R.D. (2004). Floral symmetry affects speciation rates in angiosperms. Proc. R. Soc. London. Ser. B Biol. Sci. , 271, 603-608.

Sargent, R.D. \& Ackerly, D.D. (2008). Plant-pollinator interactions and the assembly of plant communities. Trends Ecol. Evol. , 23, 123-130.

Sargent, R.D. \& Otto, S.P. (2006). The role of local species abundance in the evolution of pollinator attraction in flowering plants. Am. Nat. , 167, 67-80.

Shan, H., Cheng, J., Zhang, R., Yao, X. \& Kong, H. (2019). Developmental mechanisms involved in the diversification of flowers. Nat. Plants , 5, 917-923.

Stebbins, G.L. (1970). Adaptive radiation of reproductive characteristics in angiosperms, I: pollination mechanisms. Annu. Rev. Ecol. Syst. , 1, 307-326.

Steven, J.C., Rooney, T.P., Boyle, O.D. \& Waller, D.M. (2003). Density-dependent pollinator visitation and self-incompatibility in upper Great Lakes populations of Trillium grandiflorum . J. Torrey Bot. Soc. , 130, 23.

Thomson, J. (2003). When Is It Mutualism? Am. Nat. , 162, S1-S9.

Thomson, J.D., Wilson, P., Valenzuela, M. \& Malzone, M. (2000). Pollen presentation and pollination syndromes, with special reference toPenstemon. Plant Species Biol. , 15, 11-29.

Thorp, R.W. (2000). The collection of pollen by bees. Plant Syst. Evol. , 222, 211-223.

Thostesen, A.M. \& Olesen, J.M. (1996). Pollen removal and deposition by specialist and generalist bumblebees in Aconitum septentrionale .Oikos , 77, 77-84.

Valdovinos, F.S., Brosi, B.J., Briggs, H.M., Moisset de Espanes, P., Ramos-Jiliberto, R. \& Martinez, N.D. (2016). Niche partitioning due to adaptive foraging reverses effects of nestedness and connectance on pollination network stability. Ecol. Lett. , 19, 1277-1286.

Vamosi, J.C., Knight, T.M., Steets, J.A., Mazer, S.J., Burd, M. \& Ashman, T.L. (2006). Pollination decays in biodiversity hotspots.Proc. Natl. Acad. Sci. U. S. A. , 103, 956-961.

Vazquez, D.P. \& Aizen, M.A. (2003). Null model analyses of specialization in plant-pollinator interactions. Ecology , 84, 2493-2501.

Waser, N.M. (1986). Flower constancy: definition, cause, and measurement. Am. Nat. , 127, 593-603.

Waser, N.M., Chittka, L., Price, M. V., Williams, N.M. \& Ollerton, J. (1996). Generalization in pollination systems, and why it matters.Ecology, 77, 1043-1060.

Wolfe, L.M. \& Barrett, S.C.H. (1989). Patterns of pollen removal and deposition in tristylous Pontederia cordata L. (Pontederiaceae).Biol. J. Linn. Soc. , 36, 317-329.

Ye, Z.M., Jin, X.F., Wang, Q.F., Yang, C.F. \& Inouye, D.W. (2017). Nectar replenishment maintains the neutral effects of nectar robbing on female reproductive success of Salvia przewalskii (Lamiaceae), a plant pollinated and robbed by bumble bees. Ann. Bot. , 119, 1053-1059.

Young, H.J. \& Stanton, M.L. (1990). Influences of floral variation on pollen removal and seed production in wild radish. Ecology , 71, 536-547.

Table 1. Description of the parameters and parameter values used in the mathematical model

\begin{tabular}{ll}
\hline Parameter & Description \\
\hline$P_{t}$ & Number of pollen grains produced by the focal flower
\end{tabular}




\begin{tabular}{lll}
\hline Parameter & Description & Value \\
\hline$R_{i}$ & Proportion of pollen grains removed per visit to the focal flower by an individual of the pollinator " $i$ ". & Low \\
& Medi & High \\
$V_{i}$ & Total number of visits made by the pollinator " $i$ " in the plant community & Medi \\
& & High \\
$\Sigma A_{i} \quad$ Total floral abundance for each plant species visited by the pollinator " $i "$, excluding the focal species & Low \\
& & Medi \\
$C_{i}$ & Proportion of pollen grains carried to each subsequent flower by an individual of the pollinator " $i "$ & High \\
& & Low \\
& & Medi \\
$A_{a}$ & Abundance of the focal species & High \\
$2-150$
\end{tabular}

Table 2. Description of the parameters and parameter values used in the plant-pollinator network simulations. Equation (5) was used to determine the number of visits from each pollinator to each plant species. See Methods for justification of the values.

\begin{tabular}{llll}
\hline Parameter & Values in the different sets of simulations & Values in the different sets of simulations & Values in the different \\
\hline & & High & Intermediate high \\
$P_{t}$ & & 1000 & 1000 \\
$R_{i}$ & Max & 0.7 & 0.7 \\
& Min & 0.3 & 0.3 \\
$V_{i}$ & Average & 1500 & 1500 \\
$C_{i}$ & Max & 0.9 & 0.9 \\
& Min & 0.55 & 0.55 \\
$A_{a}$ & Average & 1000 & 500 \\
\hline
\end{tabular}

Figure 1. Importance of different pollination processes for plant pollination success as a function of floral abundance, as determined by the mathematical model. Comparison of pollination success expected for low, medium and high values of A) pollinator abundance, B) pollinator specialization, C) pollen removal and D) carryover at variable floral abundance (see Table 1 for parameter values). E) Importance of the quantity component of pollination, represented by pollinator abundance, and the quality component, determined by pollinator specialization and carryover as a function of floral abundance (measured as the average between the importance of pollinator specialization and carryover). F) Hypothetical representation of the pollination systems offering the highest pollination success at different floral abundance. Specialization on pollinators with high pollen carryover and level of specialization is favoured at low floral abundance (hypothetical examples; hawkmoths and hummingbirds). At intermediate abundance, specialized pollination by more abundant pollinators (hypothetical example; bees) is favoured. At high floral abundance, most pollinators are not sufficiently abundant to remove most pollen grains and generalization becomes more advantageous. When floral abundance is too high for the pollinator community to remove most pollen grains, reliance on abiotic pollen vectors is expected.

Figure 2. Plant-pollinator networks resulting from simulated communities of different plant species and pollinator attributes. A, B) At intermediate average flower abundance (500 flowers), plant-pollinator networks formed with plant species of variable floral abundance result in variable levels of generalization among plant species while C, D) networks formed with plant species of the same floral abundance result in similar levels of generalization. E, F) Networks composed entirely of low-abundance plant species (100 flowers) 
result in high level of specialization. G, H) Networks composed entirely of high-abundance plant species (1000 flowers) result in widespread generalization. In A, C, E and G, the thickness of the links represents the number of visits of a pollinator to a plant species. In $\mathrm{B}, \mathrm{D}, \mathrm{F}$ and $\mathrm{H}$, grey squares denote interaction between a plant and a pollinator and darker shades represent higher number of interactions.

Figure 3. Effect of interspecific variation in abundance in simulated plant communities on A) variation in degree of generalization, B) number of shared partners, C) plant-pollinator network nestedness and D) connectance at different average flower abundances. High average flower abundance corresponds to 1000, intermediate high corresponds to 500, intermediate low corresponds to 250 and low corresponds to 100. Symbols and error bars represent mean and standard error.

Figure 4. Degree of generalization and attributes of the pollinators on which a plant species colonizing simulated plant communities evolved as a function of its floral abundance. The parameter values represent the average values of pollen carryover capacity, specialization and abundance of the pollinators on which the new colonist evolved and degree of floral generalization of the new colonist. The parameter values on the $\mathrm{y}$-axis were normalized so that the minimum value corresponds to 0 and the maximum value corresponding to 1 . The standard error of the mean parameter values among the 100 simulations is presented as the shaded area around the mean values.

Figure 5. The three processes generating floral diversification according to the model. Following dispersal by a plant colonist (the red tubular flower species) from a community (represented in A) to new communities, shifts in pollination system can occur as a result of either B) change in the abundance of the new colonist, C) change in plant community composition (change in abundance of the other community members), or D) change in pollinator assemblage. C, E, G) Effect of different amounts of variation in C) abundance of the new colonist, E) community composition and G) pollinator assemblage between communities on variation in evolved pollination systems between species for simulated plant clades colonizing 20 new communities. Variation in evolved pollination systems was measured as the standard deviation of the average attributes values and degree of generalization between species of the plant clade. The parameter values on the y-axis were normalized so that the minimum value corresponds to 0 and the maximum value corresponding to 1 . Panel E) do not show values of pollinator specialization (measured as the total flower abundance of all the species it pollinates) and panel G) do not show values of pollinator abundance because those parameters were purposely varied between communities and hence variability was expected for those parameters even in the absence of shift in pollination system. Illustration by Florence Jean and Sébastien Rivest.

\section{Figure 1.}



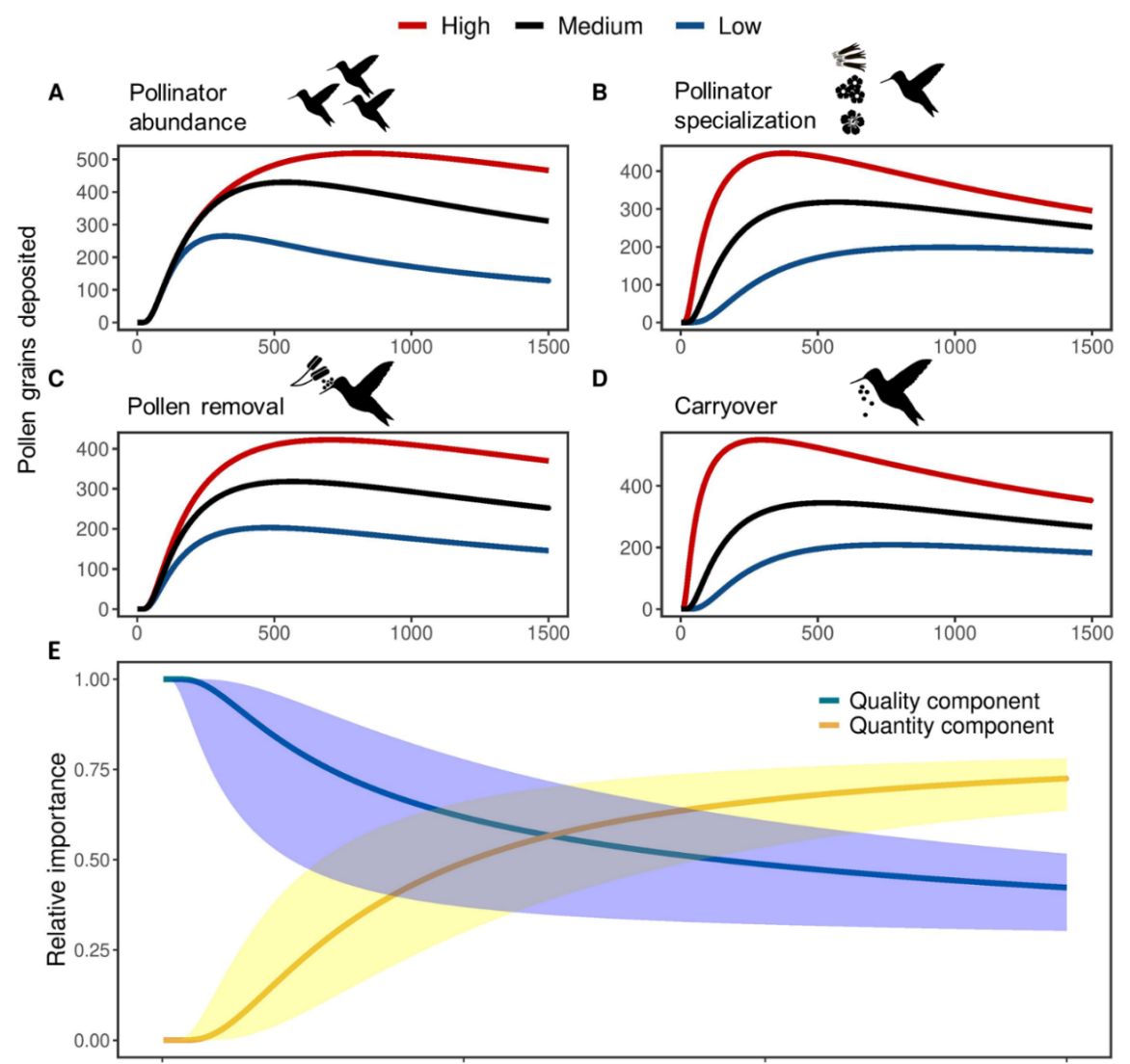

F Pollination system

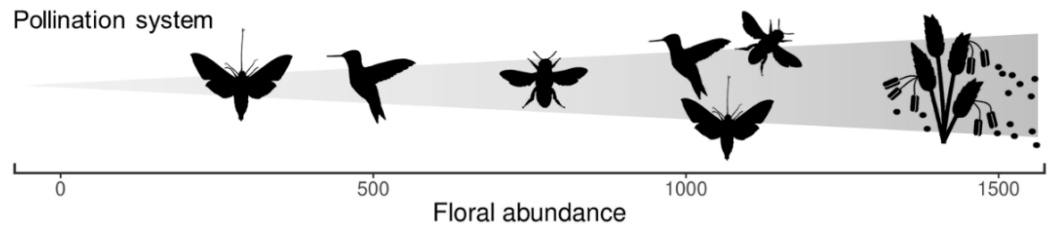

Figure 2.
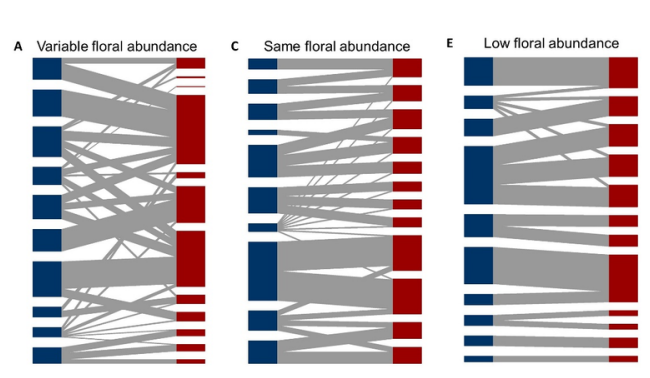

- Pollinators $\square$ Plants
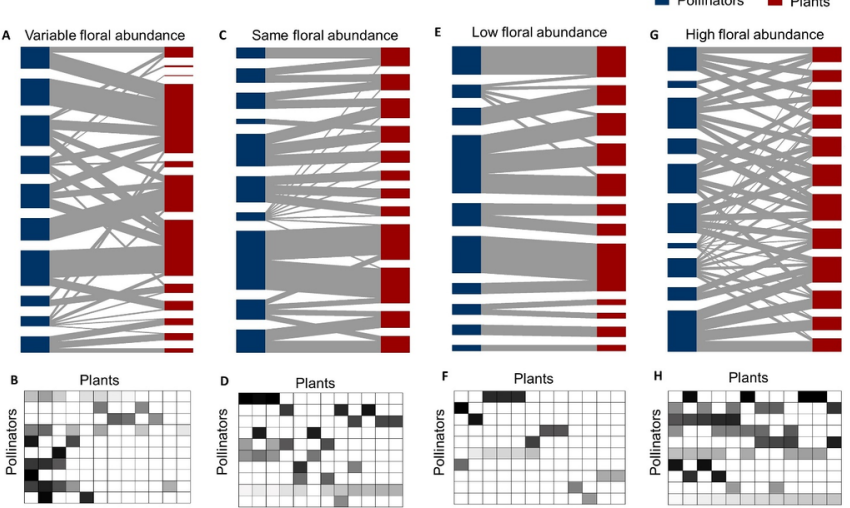
Figure 3.

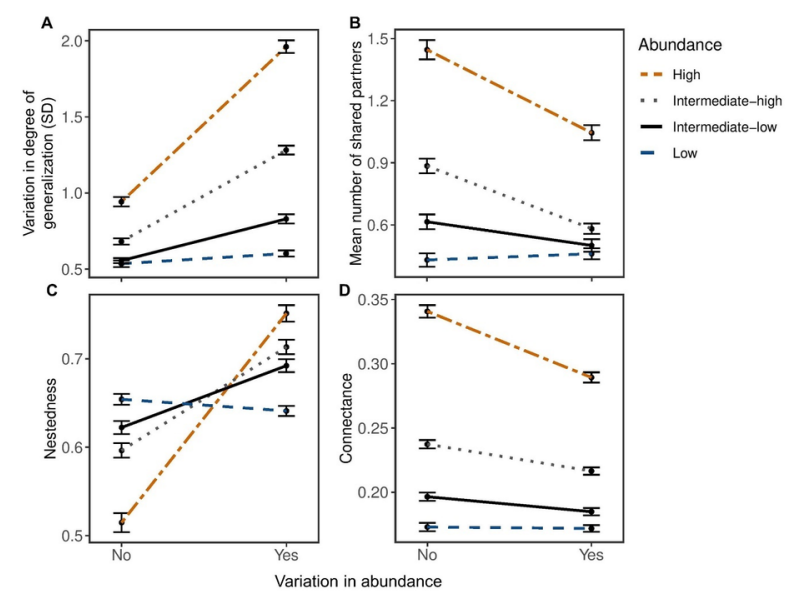

Figure 4.

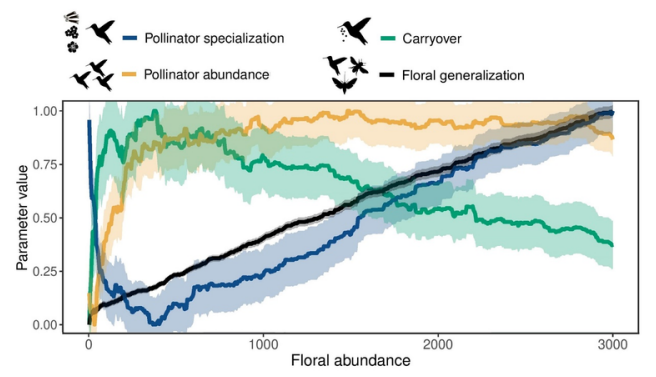

Figure 5. 


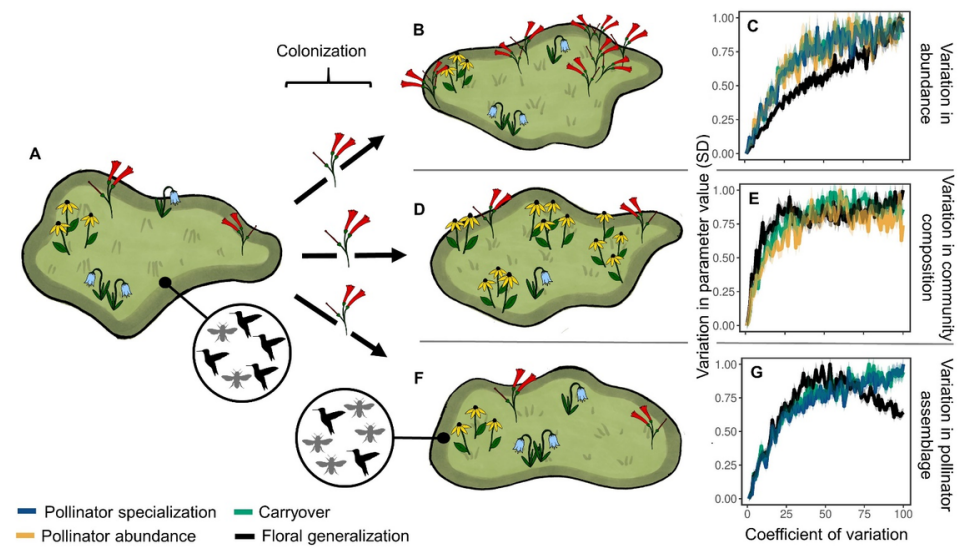

